\title{
ON CONFINEMENT INTERACTIONS IN SCALAR GENERALIZATIONS OF THE DIPOLE MODEL
}

\author{
A. Duviryak ${ }^{1}$, J. W. Darewych ${ }^{2}$ \\ ${ }^{1}$ Department for Computer Simulations of Many-Particle Systems, \\ Institute for Condensed Matter Physics of NAS of Ukraine, \\ 1, Svientsitskii St., Lviv, UA-79011, Ukraine, \\ ${ }^{2}$ Department of Physics and Astronomy, York University, \\ 4700, Keele St., Toronto, Ontario, M3J 1P3, Canada \\ (Received November 17, 2010; received in final form December 30, 2010)
}

\begin{abstract}
Two modified versions of the dipole model with a pair of mediating fields are considered. In the first version, the modification affects the Yukawa term in the Lagrangian. In the second version a non-linear self-action term is added. By constructing exact solutions of the field equations with the use of a covariant Green function we derive a Lagrangian with many-point time-nonlocal interaction terms. For the linear dipole model the two-particle interaction, in the non-relativistic limit, is shown to be a sum of Coulomb and linear confinement terms. For the nonlinear $\varphi^{3}$-model the interactions are shown to include pairwise potentials and also three-particle cluster potentials with a logarithmic confinement. The approaches towards a consistent relativistic treatment of the models are proposed, and the problem of divergences is discussed.
\end{abstract}

Key words: dipole model, confinement, potential model, quantum field theory, variational method.

PACS number(s): 11.10.Ef, 11.10.Lm

\section{INTRODUCTION}

Substantiation of confinement in QCD, both via numerical simulations $[1,2]$ and especially via analytical calculations [2], remains a challenging task. The study of simpler field-theoretical models that simulate the characteristic features of confinement remains relevant and may be useful in further investigations. The examples of such simpler models are higher derivative models and dipole models.

In the middle of the 70 s a rather simple higher derivative model [3] and a closely related dipole model [4] were proposed as a phenomenological theory of quark binding in hadrons. Subsequently, the non-Abelian version of the theory was introduced [5]. These models indicate $1 / k^{4}$ infrared asymptotics of the "gluon" propagator, and thus a linear interaction potential, even at the classical level. Unfortunately, the short-range (ultraviolet) behavior of the interaction in these models is the same, in contradiction to the $1 / k^{2}$ Coulomb-like behavior of QCD. It is of interest to modify the aforementioned models so as to adjust their properties to conform with realistic interquark interactions.

In this paper we will analyze the interactions that arise from particular generalizations of the dipole model [4]. For simplicity we consider the scalar version of the model. We modify the Yukawa term in the Lagrangian in order to take into account Coulomb-like interaction in addition to the confinement one. Another modification we shall consider involves the inclusion of nonlinear self-action terms in the mediating-field sector of the Lagrangian. As an example we shall consider the $\varphi^{3}$-nonlinearity. We show that, in the nonrelativistic approximation, this model generates a two-particle interaction and three-particle cluster interaction, both with the logarithmic-type confinement. This requires regularization of the three-point interaction potential in the static limit.

Two possible ways of the relativistic treatment of the models are considered. One is based on the classically oriented formalism of the Fokker-type action integrals. Another is a quantized field theory supplemented with the variational method. The problem of divergences of the relativistic interaction kernels and their regularization is discussed.

\section{THE NONLOCAL LAGRANGIAN FROM A SCALAR NONLINEAR DIPOLE MODEL}

We proceed from the classical action integral

$$
I=\int \mathrm{d}^{4} x \mathcal{L}(x)
$$

over Minkowski space $\left(x \in \mathbb{M}^{4}\right)$ with the Lagrangian density $(\hbar=c=1)$

$$
\mathcal{L}=\mathcal{L}_{\text {free }}+\mathcal{L}_{\mathrm{Y}}+\partial_{\mu} \chi \partial^{\mu} \varphi-\mathcal{V}(\varphi),
$$

Here $\mathcal{L}_{\text {free }}$ is the Lagrangian describing free matter. We do not need to specify it at this point (specific forms will be presented in Sections 6 and 7$)$. The $\chi(x)$ and $\varphi(x)$ are real massless scalar fields. The Yukawa term

$$
\mathcal{L}_{\mathrm{Y}}=-\rho \chi
$$

describes the interaction of the matter with the field $\chi(x) ; \rho$ is the scalar charge density of the matter. Lastly, the potential $\mathcal{V}(\varphi)$ describes the self-interaction of the $\varphi$ field; it can be chosen arbitrary.

The stationary property of the action $(2.1)-(2.3)$, i. e. $\delta I(x)=0$, determines the dynamics of the system. Thus, 
varying the action with respect to the mediating fields $\chi$ and $\varphi$ leads to the coupled set of the Euler-Lagrange equations:

$$
\begin{aligned}
& \square \varphi=-\rho, \\
& \square \chi=-\mathcal{V}^{\prime}(\varphi) .
\end{aligned}
$$

They possess the exact formal solution:

$$
\begin{aligned}
& \varphi=-D * \rho, \\
& \chi=-D * \mathcal{V}^{\prime}(\varphi),
\end{aligned}
$$

where " $*$ " denotes the convolution $[D * \rho](x) \equiv$ $\int \mathrm{d}^{4} x^{\prime} D\left(x-x^{\prime}\right) \rho\left(x^{\prime}\right)$ and $D(x)$ is a Green function of the d'Alembert equation. The arbitrary solution of the homogeneous d'Alembert equation is omitted because the free $\chi$ and $\varphi$ fields play no role in the investigation considered here.

Since the mediating fields are real, we must use a real Green function of the d'Alembert equation. The choice

$$
D_{\eta}(x)=\frac{1}{4 \pi}\left[1+\eta \operatorname{sgn} x^{0}\right] \delta\left(x^{2}\right), \quad \eta= \pm 1,0
$$

corresponds to the retarded (if $\eta=+1$ ), the advanced (if $\eta=-1$ ) or the symmetric (if $\eta=0$ ) Green function; here $x^{2} \equiv x_{\mu} x^{\mu}=\eta_{\mu \nu} x^{\mu} x^{\nu}$ and the time-like Minkowski metric $\left\|\eta_{\mu \nu}\right\|=\operatorname{diag}(+,-,-,-)$ is used. We note some useful properties of the Green functions (2.8):

$$
\begin{aligned}
& D_{\eta}(-x)=-D_{-\eta}(x), \eta= \pm 1 \\
& D_{0}(-x)=D_{0}(x)=\frac{1}{2}\left[D_{+}(x)+D_{-}(x)\right] .
\end{aligned}
$$

The solutions (2.6)-(2.7) can be substituted into the Lagrangian (2.2). The result is

$$
\overline{\mathcal{L}}=\mathcal{L}_{\text {free }}+\mathcal{L}_{\text {int }} \simeq \mathcal{L}_{\text {free }}-\mathcal{V}(-D * \rho),
$$

where $\simeq$ denotes equality modulo surface terms. This partially reduced Lagrangian depends only on the matter variables and it is non-local in space-time.

\section{A LINEAR DIPOLE MODEL}

The choice

$$
\mathcal{V}(\varphi)=\frac{1}{2} \varkappa^{2} \varphi^{2}
$$

where $\varkappa$ is an interaction constant with the dimension of mass, corresponds to the linear dipole model [6] (see also [4] where an original vector version of the linear dipole model was proposed). The interaction term of the action $I=\int \mathrm{d}^{4} x \overline{\mathcal{L}}$ with the Lagrangian density (2.10) and (3.1) implies a 2-fold integration over Minkowski space:

$$
I_{\text {int }}^{(2)}=\frac{1}{2} \varkappa^{2} \iint \mathrm{d}^{4} x \mathrm{~d}^{4} x^{\prime} \rho(x) G\left(x-x^{\prime}\right) \rho\left(x^{\prime}\right) .
$$

The Poincaré-invariant kernel of this integral is constructed by convolutions of two Green functions of d'Alembert's equation, $D_{\xi} * D_{\eta}(\xi, \eta= \pm)$, and an arbitrary additive constant. The only choice that avoids divergences in (3.2) is [6]

$$
\begin{aligned}
G(x) & =\frac{1}{2}\left[D_{+} * D_{+}+D_{-} * D_{-}\right](x)-\frac{1}{16 \pi} \\
& =\frac{1}{16 \pi}\left[\theta\left(x^{2}\right)-1\right]=-\frac{1}{16 \pi} \theta\left(-x^{2}\right) .
\end{aligned}
$$

Note that $G(x)=G(-x)$ is symmetric by construction.

In order to have some understanding of the properties of the interactions described by the non-local term (3.2) it is useful to derive a static potential, i. e., a potential of interaction between motionless point-like particles. For this purpose we take the source to be a static system of $N$ point-like particles:

$$
\rho(x)=\rho(t, \boldsymbol{x})=\sum_{a=1}^{N} g_{a} \delta\left(\boldsymbol{x}-\boldsymbol{x}_{a}\right),
$$

where $\boldsymbol{x}_{a}(a=\overline{1, N})$ is the position of the $a$-th particle and $g_{a}$ is its scalar charge. In this case we obtain:

$$
I_{\text {int }}^{(2)}=\frac{1}{2} \varkappa^{2} \sum_{a=1}^{N} \sum_{b=1}^{N} g_{a} g_{b} \int \mathrm{d} t \int \mathrm{d}^{3} x \int \mathrm{d}^{3} x^{\prime} \delta\left(\boldsymbol{x}-\boldsymbol{x}_{a}\right) \delta\left(\boldsymbol{x}^{\prime}-\boldsymbol{x}_{b}\right) \int \mathrm{d} t^{\prime} G\left(x-x^{\prime}\right) \equiv-\sum_{a} \sum_{b} \int \mathrm{d} t V\left(\boldsymbol{x}_{a}-\boldsymbol{x}_{b}\right) .
$$

It is obvious that the function

$$
V\left(\boldsymbol{x}_{a}-\boldsymbol{x}_{b}\right)=-\varkappa^{2} g_{a} g_{b} \int \mathrm{d} t^{\prime} G\left(t^{\prime}, \boldsymbol{x}_{a}-\boldsymbol{x}_{b}\right)=\frac{\varkappa^{2} g_{a} g_{b}}{8 \pi}\left|\boldsymbol{x}_{a}-\boldsymbol{x}_{b}\right|
$$

is a static linearly confining potential.

The above dipole model does not include short-range Coulomb-like interactions, as would arise in realistic descriptions of inter-quark forces. Such a Coulombic interaction can be easily generated by modifying the Yukawa term (2.3) in the Lagrangian (2.2) as follows:

$$
\mathcal{L}_{\mathrm{Y}} \rightarrow \tilde{\mathcal{L}}_{\mathrm{Y}}=-\rho\left(\chi+\frac{1}{2} \varphi\right) .
$$

As in the case of Eqs. (2.4)-(2.5), the modified field equations are easily solvable, and the corresponding reduced Lagrangian has the form:

$$
\overline{\mathcal{L}}=\mathcal{L}_{\text {free }}+\frac{1}{2} \rho D * \rho+\mathcal{V}(-D * \rho) .
$$

For the present $\varphi^{2}$-interaction case, Eq. (3.1), the action 
integral (3.2) with (3.3) modifies to the form

$$
I_{\text {int }}^{(2)}=\frac{1}{2} \iint \mathrm{d}^{4} x \mathrm{~d}^{4} x^{\prime} \rho(x)\left\{\varkappa^{2} G\left(x-x^{\prime}\right)-D_{0}\left(x-x^{\prime}\right)\right\} \rho\left(x^{\prime}\right),
$$

which leads, in the static limit, to the "funnel" (or "Cornell") two-particle potential:

$$
V\left(\boldsymbol{x}_{1}-\boldsymbol{x}_{2}\right)=\frac{g_{a} g_{b}}{4 \pi}\left\{\frac{\varkappa^{2}}{2}\left|\boldsymbol{x}_{1}-\boldsymbol{x}_{2}\right|-\frac{1}{\left|\boldsymbol{x}_{1}-\boldsymbol{x}_{2}\right|}\right\} .
$$

We note that the static potentials (3.6) and (3.10) actually correspond to a non-relativistic (i.e., slow motion) approximation of an interaction in a system of point-like particles. Indeed, let us regard the particle positions in (3.4) as slowly varying functions of $t: \boldsymbol{x}_{a}=\boldsymbol{x}_{a}(t)$, such that $v_{a} \equiv\left|\mathrm{d} \boldsymbol{x}_{a} / \mathrm{d} t\right| \ll 1$. In this case the expression (3.4) is approximately equal to the manifestly covariant charge density (6.2) considered in Section 6. Accordingly, equality (3.5) holds approximately, up to negligibly small (quasi-relativistic) corrections $\propto v_{a}^{2}$. Thus these static potentials are appropriate for use in non-relativistic potential models of hadrons. This topic will be discussed in Section 5 .

\section{THE $\varphi^{3}$-NONLINEAR DIPOLE MODEL}

The simplest non-linear version of the dipole model is that with a cubic self-action potential:

$$
\mathcal{V}(\varphi)=\frac{1}{3} \kappa \varphi^{3}
$$

where $\kappa$ is an interaction constant with the dimensions of mass. Then, the corresponding interaction term in the non-local action integral has the form:

$$
\begin{aligned}
I_{\text {int }}^{(3)} & =\frac{1}{3} \kappa \iiint \int \mathrm{d}^{4} x \mathrm{~d}^{4} x^{\prime} \mathrm{d}^{4} x^{\prime \prime} \mathrm{d}^{4} x^{\prime \prime \prime} D\left(x-x^{\prime}\right) D\left(x-x^{\prime \prime}\right) D\left(x-x^{\prime \prime \prime}\right) \rho\left(x^{\prime}\right) \rho\left(x^{\prime \prime}\right) \rho\left(x^{\prime \prime \prime}\right) \\
& \equiv \frac{1}{3} \kappa \iiint \mathrm{d}^{4} x^{\prime} \mathrm{d}^{4} x^{\prime \prime} \mathrm{d}^{4} x^{\prime \prime \prime} F\left(x^{\prime}, x^{\prime \prime}, x^{\prime \prime \prime}\right) \rho\left(x^{\prime}\right) \rho\left(x^{\prime \prime}\right) \rho\left(x^{\prime \prime \prime}\right) .
\end{aligned}
$$

This expression is rather symbolic until the choice of Green functions $D$ (and thus the kernel $F$ ) is specified. To this end, let us consider the convolution of three arbitrary chosen Green functions:

$F_{\xi \eta \zeta}\left(x_{1}, x_{2}, x_{3}\right)=\int \mathrm{d}^{4} z D_{\xi}\left(z-x_{1}\right) D_{\eta}\left(z-x_{2}\right) D_{\zeta}\left(z-x_{3}\right)$.

This function possesses the following formal properties:

1. translational invariance:

$F_{\xi \eta \zeta}\left(x_{1}+\lambda, x_{2}+\lambda, x_{3}+\lambda\right)=F_{\xi \eta \zeta}\left(x_{1}, x_{2}, x_{3}\right)$, where $\lambda \in \mathbb{M}^{4}$

2. Lorentz invariance:

$F_{\xi \eta \zeta}\left(\Lambda x_{1}, \Lambda x_{2}, \Lambda x_{3}\right)=F_{\xi \eta \zeta}\left(x_{1}, x_{2}, x_{3}\right)$, where $\Lambda \in$ $\mathrm{SO}(1,3)$

3. inversional property: $F_{\xi \eta \zeta}\left(-x_{1},-x_{2},-x_{3}\right)=F_{-\xi-\eta-\zeta}\left(x_{1}, x_{2}, x_{3}\right)$.

4. permutational properties:

$$
\begin{aligned}
& F_{\eta \xi \zeta}\left(x_{2}, x_{1}, x_{3}\right)=F_{\zeta \xi \eta}\left(x_{3}, x_{1}, x_{2}\right)=\cdots \\
& =F_{\xi \eta \zeta}\left(x_{1}, x_{2}, x_{3}\right)
\end{aligned}
$$

It follows from property 1 that function (4.3) actually depends on two linearly independent 4-vector arguments only; for example,

$$
F_{\xi \eta \zeta}\left(x_{1}, x_{2}, x_{3}\right) \equiv F_{\xi \eta \zeta}\left(x_{1}-x_{3}, x_{2}-x_{3}\right) .
$$

In addition, because of properties 3 and 4 , all possible functions $F_{\xi \eta \zeta}$ can be expressed, in terms of only two functions $F_{-++}$and $F_{+++}$. These functions are calculated in the Appendix with the use of property 2:

$$
\begin{aligned}
& F_{-++}(u, v)=\frac{\theta\left(u^{0}\right) \theta\left(u^{2}\right) \theta\left(-v^{2}\right) \theta\left[-(u-v)^{2}\right]}{2(4 \pi)^{2} \sqrt{(u \cdot v)^{2}-u^{2} v^{2}}}, \\
& F_{+++}(u, v)=\left\{\begin{array}{cc}
\infty, & \text { if } u \& v \text { are space-like } \\
0 & \text { otherwise }
\end{array}\right.
\end{aligned}
$$

The function $F_{+++}$is divergent, hence only the function $F_{-++}$can be used in the action integral (4.2).

Since the kernel $F\left(x_{1}, x_{2}, x_{3}\right)$ of the action integral (4.2) is completely symmetric by construction, it must be the inversion invariant (cf. property 3 ). The only choice that ensures this property is:

$$
\begin{aligned}
F\left(x_{1}, x_{2}, x_{3}\right) & =\frac{1}{6}\left[F_{-++}\left(x_{1}, x_{2}, x_{3}\right)+F_{-++}\left(x_{2}, x_{1}, x_{3}\right)+F_{-++}\left(x_{3}, x_{1}, x_{2}\right)+F_{-++}\left(-x_{1},-x_{2},-x_{3}\right)\right. \\
& \left.+F_{-++}\left(-x_{2},-x_{1},-x_{3}\right)+F_{-++}\left(-x_{3},-x_{1},-x_{2}\right)\right]
\end{aligned}
$$


In the static approximation the choice of the Green function is not important and need not be specified explicitly. In this case we have

$$
\begin{aligned}
I_{\text {int }}^{(3)} & =\frac{1}{3} \kappa \int \mathrm{d} t^{\prime} \int \mathrm{d}^{3} x^{\prime} \int \mathrm{d}^{3} x^{\prime \prime} \int \mathrm{d}^{3} x^{\prime \prime \prime} \rho\left(\boldsymbol{x}^{\prime}\right) \rho\left(\boldsymbol{x}^{\prime \prime}\right) \rho\left(\boldsymbol{x}^{\prime \prime \prime}\right) \\
& \times \int \mathrm{d} t^{\prime \prime} \int \mathrm{d} t^{\prime \prime \prime} \int \mathrm{d}^{4} x D\left(x-x^{\prime}\right) D\left(x-x^{\prime \prime}\right) D\left(x-x^{\prime \prime \prime}\right) \\
& =-\frac{\kappa}{3(4 \pi)^{3}} \int \mathrm{d} t \int \mathrm{d}^{3} x^{\prime} \int \mathrm{d}^{3} x^{\prime \prime} \int \mathrm{d}^{3} x^{\prime \prime \prime} \rho\left(\boldsymbol{x}^{\prime}\right) \rho\left(\boldsymbol{x}^{\prime \prime}\right) \rho\left(\boldsymbol{x}^{\prime \prime \prime}\right) \\
& \times U\left(\boldsymbol{x}^{\prime}, \boldsymbol{x}^{\prime \prime}, \boldsymbol{x}^{\prime \prime \prime}\right),
\end{aligned}
$$

where the kernel

$$
U\left(\boldsymbol{x}_{1}, \boldsymbol{x}_{2}, \boldsymbol{x}_{3}\right)=-\int \frac{\mathrm{d}^{3} z}{\left|\boldsymbol{z}-\boldsymbol{x}_{1}\right|\left|\boldsymbol{z}-\boldsymbol{x}_{2}\right|\left|\boldsymbol{z}-\boldsymbol{x}_{3}\right|}
$$

has the structure (modulo a constant factor) of a threeparticle interaction potential.

The integral in r.h.s. of (4.9) is a divergent quantity and thus it may seem to be meaningless. However, the gradients $\partial U\left(\boldsymbol{x}_{1}, \boldsymbol{x}_{2}, \boldsymbol{x}_{3}\right) / \partial \boldsymbol{x}_{a}(a=1,2,3)$ which determine the forces in the classical background of this problem, are well defined and finite. Thus the "function"(4.9) can be presented in the form

$$
U\left(\boldsymbol{x}_{1}, \boldsymbol{x}_{2}, \boldsymbol{x}_{3}\right)=\tilde{U}\left(\boldsymbol{x}_{1}, \boldsymbol{x}_{2}, \boldsymbol{x}_{3}\right)+U_{0}
$$

where $\tilde{U}\left(\boldsymbol{x}_{1}, \boldsymbol{x}_{2}, \boldsymbol{x}_{3}\right)$ is a regular (finite) function and $U_{0}$ is a physically unimportant infinite negative constant (independent of the variables $\left.\boldsymbol{x}_{1}, \boldsymbol{x}_{2}, \boldsymbol{x}_{3}\right)$.

In order to perform this replacement and calculate $\tilde{U}$ explicitly, let us first list some general symmetry properties of integral (4.9) (which are related to properties of the kernel $F(4.7))$ :

1. translational invariance: $U\left(\boldsymbol{x}_{1}+\boldsymbol{\lambda}, \boldsymbol{x}_{2}+\boldsymbol{\lambda}, \boldsymbol{x}_{3}+\boldsymbol{\lambda}\right)=U\left(\boldsymbol{x}_{1}, \boldsymbol{x}_{2}, \boldsymbol{x}_{3}\right)$, where $\lambda \in \mathbb{R}^{3}$;

2. rotational invariance:

$U\left(\mathrm{R} \boldsymbol{x}_{1}, \mathrm{R} \boldsymbol{x}_{2}, \mathrm{R} \boldsymbol{x}_{3}\right)=U\left(\boldsymbol{x}_{1}, \boldsymbol{x}_{2}, \boldsymbol{x}_{3}\right)$, where $\mathrm{R} \in$ $\mathrm{SO}(3)$;
3. inversional invariance: $U\left(-\boldsymbol{x}_{1},-\boldsymbol{x}_{2},-\boldsymbol{x}_{3}\right)=$ $U\left(\boldsymbol{x}_{1}, \boldsymbol{x}_{2}, \boldsymbol{x}_{3}\right)$.

4. permutational invariance:

$$
U\left(\boldsymbol{x}_{2}, \boldsymbol{x}_{1}, \boldsymbol{x}_{3}\right)=U\left(\boldsymbol{x}_{1}, \boldsymbol{x}_{3}, \boldsymbol{x}_{2}\right)=U\left(\boldsymbol{x}_{1}, \boldsymbol{x}_{2}, \boldsymbol{x}_{3}\right) ;
$$

These properties are fundamental symmetries inherent to any interaction potential of a closed (nonrelativistic) system of three particles with identical interaction properties. Thus the regularized potential must possess these properties with necessity. As a consequence, the potential $\tilde{U}\left(\boldsymbol{x}_{1}, \boldsymbol{x}_{2}, \boldsymbol{x}_{3}\right)$ actually depends only on the three inter-point distances $x_{12}, x_{13}, x_{23}$, where $x_{a b}=\left|\boldsymbol{x}_{a b}\right| \equiv$ $\left|\boldsymbol{x}_{a}-\boldsymbol{x}_{b}\right|$.

In an earlier paper [7] a representation of function (4.9) was given in which its dependence on the inter pair distances is manifest. This representation simplifies considerably the regularization and evaluation of $U$. It is based on an application of the well known formula:

$$
\frac{1}{r}=\frac{1}{\sqrt{\pi}} \int_{-\infty}^{\infty} \mathrm{d} k \mathrm{e}^{-k^{2} r^{2}}
$$

to each Coulomb-like factor of the integrand of the expression (4.9), which thereby takes the form of the 6 -fold integral $\int \mathrm{d}^{3} k \int \mathrm{d}^{3} z \ldots$ The integrand is Gaussian with respect to the variable $\boldsymbol{z}$ and thus the integration can be carried out easily. As a result, the dependence of the potential $U\left(x_{12}, x_{23}, x_{13}\right)$ on the three inter pair distances $x_{a b}$ becomes apparent.

The potential difference:

$$
\begin{aligned}
& U\left(x_{12}, x_{23}, x_{13}\right)-U\left(y_{12}, y_{23}, y_{13}\right) \\
& =\tilde{U}\left(x_{12}, x_{23}, x_{13}\right)-\tilde{U}\left(y_{12}, y_{23}, y_{13}\right)
\end{aligned}
$$

must be finite since infinite constants $U_{0}$ (see (4.10)) from the first and second terms of (4.11) mutually cancel out. The l.h.s. of (4.11) can be calculated explicitly and the result reduced to a quadrature [7]:

$$
\tilde{U}\left(\boldsymbol{x}_{1}, \boldsymbol{x}_{2}, \boldsymbol{x}_{3}\right) \equiv \tilde{U}\left(x_{12}, x_{23}, x_{13}\right)=4 \pi \ln \frac{x_{13}+x_{23}}{4 a}+I(\alpha, \beta),
$$

where

$$
\begin{aligned}
& I(\alpha, \beta)=4 \int_{-1}^{1} \frac{\mathrm{d} s}{\sqrt{(s+\alpha)^{2}+\beta^{2}}} \arctan \sqrt{\frac{(\mathrm{s}+\alpha)^{2}+\beta^{2}}{1-\mathrm{s}^{2}}}, \\
& \alpha=\frac{x_{13}^{2}-x_{23}^{2}}{x_{12}^{2}}, \quad \beta^{2}=\frac{\left[\left(x_{13}+x_{23}\right)^{2}-x_{12}^{2}\right]\left[x_{12}^{2}-\left(x_{13}-x_{23}\right)^{2}\right]}{x_{12}^{4}},
\end{aligned}
$$

and $a$ is an arbitrary constant with dimensions of length. We note that the interparticle distances must satisfy the triangle inequalities: $x_{13}+x_{23} \geq x_{12}, x_{23}+x_{12} \geq x_{13}$ and $x_{12}+x_{13} \geq x_{23}$

The regularized potential (4.12)-(4.14) possesses all properties ennumerated above, including the permuta- 
tional invariance (although implicitly). In addition, it obeys the following:

5. scaling property: $\tilde{U}\left(\lambda \boldsymbol{x}_{1}, \lambda \boldsymbol{x}_{2}, \lambda \boldsymbol{x}_{3}\right)=$ $\tilde{U}\left(\boldsymbol{x}_{1}, \boldsymbol{x}_{2}, \boldsymbol{x}_{3}\right)+4 \pi \ln \lambda$, where $\lambda \in \mathbb{R}_{+}$.

In a particular case when the points $\boldsymbol{x}_{1}, \boldsymbol{x}_{2}$ and $\boldsymbol{x}_{3}$ lie on a straight line the integral (4.13) can be calculated analytically:

$$
\begin{aligned}
& \tilde{U}\left(x_{12}, x_{23}, x_{13}\right)=4 \pi \ln \frac{x_{>}}{2 a}, \\
& \text { where } \quad x_{>}=\max \left(x_{12}, x_{23}, x_{13}\right) .
\end{aligned}
$$

This case includes configurations where two of three points coincide, say, $\boldsymbol{x}_{1}=\boldsymbol{x}_{3}$; i.e., $x_{13}=0$, and $x_{>}=$ $x_{12}=x_{23}$ in r.h.s. of (4.15).

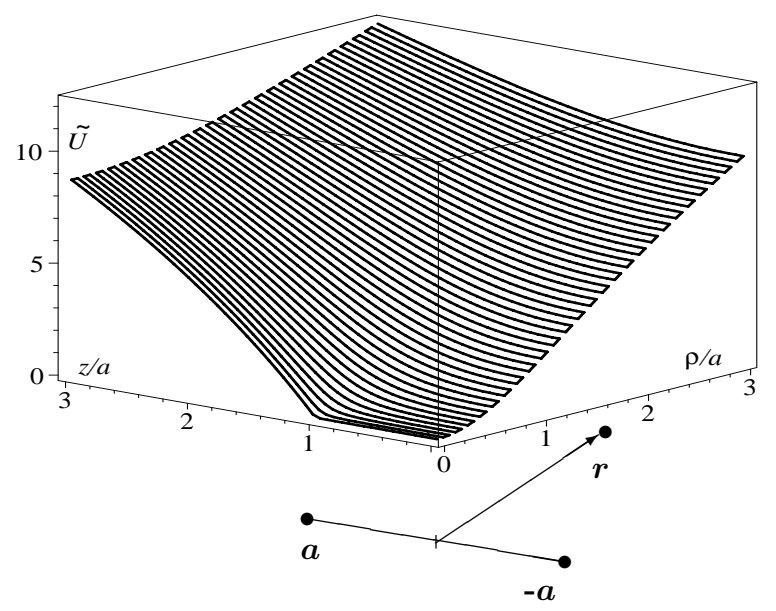

Fig. 1. The potential $\tilde{U}(\boldsymbol{a},-\boldsymbol{a}, \boldsymbol{r})$ as a function of $\boldsymbol{r}=\{x, y, z\} ; \rho=\sqrt{x^{2}+y^{2}} ; a=|\boldsymbol{a}|$. The function is symmetric under the inversion $z \rightarrow-z$ and rotation around $0 z$. In particular, $\tilde{U}=4 \pi \theta(|z|-a) \ln \frac{1}{2}(|z| / a+1)$ if $\rho=0$.

Another analytically solvable case is that of equidistant separations $x_{12}=x_{23}=x_{13}=r$, for which

$$
\tilde{U}(r)=4 \pi \ln \frac{r}{a}+c_{1},
$$

where $a$ is any convenient unit of length and $c_{1}$ is a (physically irrelevant) constant.

In the general case, a numerical integration of (4.13) is required. We illustrate the behavior of the potential in Figure 1 for the particular case $\boldsymbol{x}_{1}=\boldsymbol{a}, \boldsymbol{x}_{2}=-\boldsymbol{a}$ as a function of $\boldsymbol{x}_{3}=\boldsymbol{r}$. The value of potential for an arbitrary configuration can be obtained from this using the symmetry properties $\mathbf{1}-\mathbf{5}$.

In the case when one of the points is far from the others equality (4.15) is valid asymptotically. Thus the regularized potential reveals logarithmic confinement properties.

\section{THE APPLICATION TO NON-RELATIVISTIC POTENTIAL MODELS}

Generalized dipole models may have an application to the spectroscopy of hadrons. The funnel potential with a linearly rising long-range interaction (3.10), which we derived here from the $\varphi^{2}$-model, has been used successfully in potential models of heavy mesons; see [8] and refs. therein. The funnel potential has also been applied successfully to the description of baryons [9]. Due to the linearity of the $\varphi^{2}$-model, the three-particle interaction in this case is a superposition of pair-wise potentials.

The $\varphi^{3}$-nonlinear dipole model reveals a different character of confinement. Let us derive two- and threeparticle interaction potentials for this model in the nonrelativistic approximation. To this end we insert the point-like density (3.4) into action (4.8) (with the regularized kernel $\tilde{U}$ ) and represent the latter in the form:

$$
I_{\mathrm{int}}^{(3)}=-\int \mathrm{d} t V
$$

where

$$
\begin{aligned}
V & =\frac{\kappa}{3(4 \pi)^{3}} \sum_{a} \sum_{b} \sum_{c} g_{a} g_{b} g_{c} \tilde{U}\left(x_{a b}, x_{b c}, x_{c a}\right) \\
& =\frac{\kappa}{3(4 \pi)^{3}}\left\{\sum_{a} g_{a}^{3} \tilde{U}(0,0,0)+3 \sum_{a<b} \sum_{a} g_{a} g_{b}\left(g_{a}+g_{b}\right) \tilde{U}\left(x_{a b}, x_{a b}, 0\right)+6 \sum_{a<b<c} \sum_{c} g_{a} g_{b} g_{c} \tilde{U}\left(x_{a b}, x_{b c}, x_{c a}\right)\right\}
\end{aligned}
$$

The terms containing the infinite constant $\tilde{U}(0,0,0)$ correspond to self-interaction energy and should be omitted. The pairwise terms contribute the two-body potential which, taking account of eq. (4.15) and the remark following this equation, takes the form:

$$
V\left(\boldsymbol{x}_{1}-\boldsymbol{x}_{2}\right)=\frac{\kappa g_{1} g_{2}\left(g_{1}+g_{2}\right)}{(4 \pi)^{2}} \ln \frac{x_{12}}{2 a} \text {. }
$$

The logarithmic funnel-shaped potential is qualitatively similar to the potential (3.10); see Figure 2. It is also used in potential models of mesons [8].

In the three-body potential, the cluster term $\tilde{U}\left(\boldsymbol{x}_{1}, \boldsymbol{x}_{2}, \boldsymbol{x}_{3}\right)$, eqs. (4.12)-(4.14), arises along with the pairwise terms: 
$V\left(\boldsymbol{x}_{1}, \boldsymbol{x}_{2}, \boldsymbol{x}_{3}\right)=\frac{\kappa}{(4 \pi)^{2}}\left\{g_{1} g_{2}\left(g_{1}+g_{2}\right) \ln \frac{x_{12}}{2 a}+g_{2} g_{3}\left(g_{2}+g_{3}\right) \ln \frac{x_{23}}{2 a}+g_{3} g_{1}\left(g_{3}+g_{1}\right) \ln \frac{x_{31}}{2 a}\right\}+\frac{2 \kappa g_{1} g_{2} g_{3}}{(4 \pi)^{3}} \tilde{U}\left(\boldsymbol{x}_{1}, \boldsymbol{x}_{2}, \boldsymbol{x}_{3}\right)$

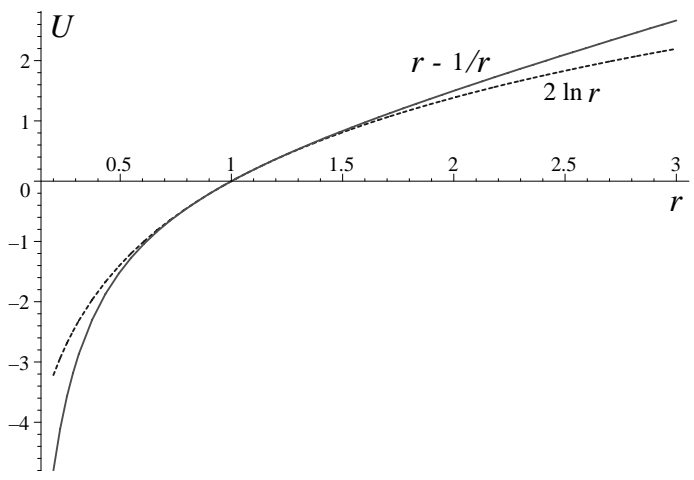

Fig. 2. Comparison of the Cornell and logarithmic potentials.

The breaking of the superposition principle is caused by the nonlinearity of the model.

The use of logarithmic funnel potentials in phenomenological models of hadrons has some advantages. For example, in the two-particle problem with such a potential, the differences between energy levels do not depend on rest masses of the particles, and such behaviour is observed experimentally [8].

The study of properties of three-particle systems in models with logarithmically rising potential requires the solution and analysis of the three-body Schrödinger equation. This is a challenging task, which will not be undertaken in the present work.

\section{A RELATIVISTIC TREATMENT: THE FOKKER-TYPE FORMALISM}

The non-relativistic treatment of the dipole models revealed their confinement properties. Now we return to a more consistent relativistic description which, up to this point, is not as yet complete. We need to specify a dynamics of the matter subsystem of the models. This can be done in several ways. In the present work, we consider two distinct approaches; one is classical-oriented and the other is a QFT approach. Both give rise to some difficulties, which we outline briefly.
Within the classical framework, matter is considered to be a system of $N$ point-like particles. It is characterized by the following free-particle Lagrangian and charge density:

$$
\begin{gathered}
\mathcal{L}_{\text {free }}(x)=-\sum_{a} m_{a} \int \mathrm{d} s_{a} \delta\left(x-z_{a}\left(\tau_{a}\right)\right) ; \\
\rho(x)=\sum_{a} g_{a} \int \mathrm{d} s_{a} \delta\left(x-z_{a}\left(\tau_{a}\right)\right) .
\end{gathered}
$$

Here $m_{a}$ and $g_{a}$ are the rest mass and the charge of the $a$-th particle; $z_{a}^{\mu}\left(\tau_{a}\right)(\mu=\overline{0,3}, a=\overline{1, N})$ are the covariant coordinates of the world line of the $a$-th particle, parameterized by an arbitrary evolution parameter $\tau_{a}$ (the proper time $s_{a}$ is used frequently); $d s_{a} \equiv \sqrt{\dot{z}_{a}^{2}} d \tau_{a}$; $\dot{z}_{a}^{\mu}\left(\tau_{a}\right) \equiv d z_{a}^{\mu} / d \tau_{a}$.

The substitution of (6.1) into action (2.1), and of (6.2) into action (3.2) of the modified linear dipole model leads to the Fokker-type action integral [10,11]:

$$
\begin{aligned}
I= & I_{\text {free }}+I_{\text {int }}^{(2)} \equiv-\sum_{a} m_{a} \int \mathrm{d} s_{a} \\
& +\sum_{a<b} \sum_{<} g_{a} g_{b} \iint \mathrm{d} s_{a} \mathrm{~d} s_{b}\left\{\varkappa^{2} G\left(z_{a b}\right)-D_{0}\left(z_{a b}\right)\right\},
\end{aligned}
$$

where $z_{a b}^{\mu} \equiv z_{a}^{\mu}\left(\tau_{a}\right)-z_{b}^{\mu}\left(\tau_{b}\right)$. This expression is welldefined and regular by provided that, in the second (double) integral, the divergent self-interaction terms corresponding to coincident particle indices $a=b$ are removed from the sum.

The variation of the particle variables $z_{a}^{\mu}\left(\tau_{a}\right)$ in action (6.3) gives rise to integro-differential equations of motion which complicate greatly the analysis of the model, even in the simplest two-particle case. The transition to the Hamiltonian formalism and quantization cannot be performed directly. Similar two-particle Fokker-type models and some approximation methods appropriate for the Hamiltonian description, and the quantization of those and the present models are considered in [12-16].

In the case of the $\varphi^{3}$-nonlinear model action (4.2) is given by the three-fold Fokker integral:

$$
\begin{aligned}
I_{\text {int }}^{(3)} & =\frac{1}{3} \kappa \sum_{a} \sum_{b} \sum_{c}^{\prime} g_{a} g_{b} g_{c} \iiint \mathrm{d} s_{a} \mathrm{~d} s_{b} \mathrm{~d} s_{c} F\left(z_{a}, z_{b}, z_{c}\right) \\
& =\kappa \sum_{a \neq b} \sum_{b} g_{a}^{2} g_{b} \iiint \mathrm{d} s_{a} \mathrm{~d} s_{a}^{\prime} \mathrm{d} s_{b} F\left(z_{a}, z_{a}^{\prime}, z_{b}\right)+2 \kappa \sum_{a<b} \sum_{b<c} g_{a} g_{b} g_{c} \iiint \mathrm{d} s_{a} \mathrm{~d} s_{b} \mathrm{~d} s_{c} F\left(z_{a}, z_{b}, z_{c}\right),
\end{aligned}
$$


where $z_{a}^{\prime} \equiv z_{a}\left(\tau_{a}^{\prime}\right), s_{a}^{\prime} \equiv s_{a}\left(\tau_{a}^{\prime}\right)$ and the "backprimed" summa skips terms corresponding to $a=b=c$. In contrast to action (6.3) of the linear model, this action is not well defined. Even if the self-interaction terms are omitted, the integrands $F\left(z_{a}, z_{a}^{\prime}, z_{b}\right)$ of the pair-wise terms become ill defined as the integration variable $\tau_{a}^{\prime}$ approaches $\tau_{a}$, given definitions (4.3)-(4.5), (4.7). In this particular case a more subtle analysis of the integral (4.3) is necessary. Also, it must be remembered that the non-relativistic three-particle potential (4.9) is divergent. Thus the integrands F's in the relativistic action (6.4) are not integrable and thus should be regularized. We shall not consider this problem further in this work.

\section{A RELATIVISTIC TREATMENT: THE QFT VARIATIONAL METHOD}

In this section we consider a field-theoretical treatment of matter. Matter is, more commonly, represented by a system of fermionic fields. However, in this work, we will consider it to be the complex scalar field.

We proceed from the Lagrangian and charge density:

$$
\mathcal{L}_{\text {free }}=\partial_{\mu} \phi^{*} \partial^{\mu} \phi-m^{2} \phi^{*} \phi, \quad \rho=g \phi^{*} \phi,
$$

where $\phi(x)$ is a complex scalar "matter" field with rest mass $m$ and charge $g$.

The next step is a transition to the Hamiltonian formalism. The procedure is rather complex, due to a nonlocality of the Lagrangian description. It can be performed perturbatively, following [6]. In the leading-order approximation the procedure reduces to the following formal prescription $[6,7,17-19]$. We construct the Hamiltonian density,

$$
\mathcal{H}=\mathcal{H}_{\text {free }}-\mathcal{L}_{\text {int }}
$$

where $\mathcal{H}_{\text {free }}$ is the standard expression, and $\mathcal{L}_{\text {int }}$ is specified by (2.10) or (3.8). It is then expressed in terms of the Fourier amplitudes $A_{\mathbf{k}}, B_{\mathbf{k}}$ and $A_{\mathbf{k}}^{\dagger}, B_{\mathbf{k}}^{\dagger}$ of the field $\phi(x)$ (see Eq. (2.14) in [20]). Upon quantization these amplitudes satisfy the standard commutation relations and become the creation and annihilation operators. Then, the canonical normal-ordered Hamiltonian operator is given by $H=\int \mathrm{d}^{3} x: \mathcal{H}(t=0, \boldsymbol{x}):$.

Since the QFT eigenvalue equation $H|\Psi\rangle=E|\psi\rangle$ is not solvable, we use the variational approximation $\delta\left\langle\Psi_{\mathrm{tr}}|H-E| \Psi_{\mathrm{tr}}\right\rangle=0$. The trial state of the system, $\left|\Psi_{\mathrm{tr}}\right\rangle$, is built of few particle channel components such as the two-particle state vector $|2\rangle=\frac{1}{\sqrt{2}} \int \mathrm{d}^{3} p_{1} \mathrm{~d}^{3} p_{2} f\left(\boldsymbol{p}_{1}, \boldsymbol{p}_{3}\right) A_{\boldsymbol{p}_{1}}^{\dagger} A_{\boldsymbol{p}_{2}}^{\dagger}|0\rangle$, the particle-antiparticle vector $|1+\overline{1}\rangle=\int \mathrm{d}^{3} p_{1} \mathrm{~d}^{3} p_{2} f\left(\boldsymbol{p}_{1}, \boldsymbol{p}_{3}\right)$ $\times A_{\boldsymbol{p}_{1}}^{\dagger} B_{\boldsymbol{p}_{2}}^{\dagger}|0\rangle$, the three-particle one $|3\rangle=\frac{1}{\sqrt{3 !}}$ $\times \int \mathrm{d}^{3} p_{1} \mathrm{~d}^{3} p_{2} \mathrm{~d}^{3} p_{3} f\left(\boldsymbol{p}_{1}, \boldsymbol{p}_{2}, \boldsymbol{p}_{3}\right) A_{\boldsymbol{p}_{1}}^{\dagger} A_{\boldsymbol{p}_{2}}^{\dagger} A_{\boldsymbol{p}_{3}}^{\dagger}|0\rangle$, etc. To implement the variational approximation, we evaluate the matrix elements of $H$.

In the case of the linear dipole model the interaction Lagrangian $\mathcal{L}_{\text {int }} \equiv \mathcal{L}_{\text {int }}^{(2)}$ (and thus the Hamiltonian $\left.H_{\text {int }}^{(2)}\right)$ is a bilinear functional of the charge density $\rho$. Accordingly, the matrix elements $\left\langle 2\left|H_{\text {int }}^{(2)}\right| 2\right\rangle$ and $\left\langle 1+\overline{1}\left|H_{\text {int }}^{(2)}\right| 1+\overline{1}\right\rangle$ are the relevant ones for two-particle and particle-antiparticle variational problems. They lead to a variational Salpeter-like wave equations of the form:

$$
\begin{aligned}
& \left\{p_{10}+p_{20}-E\right\} f\left(\boldsymbol{p}_{1}, \boldsymbol{p}_{2}\right) \\
& \quad+\int \mathrm{d}^{3} p_{1}^{\prime} \mathrm{d}^{3} p_{2}^{\prime} \mathcal{K}^{(2)}\left(\boldsymbol{p}_{1}, \boldsymbol{p}_{2}, \boldsymbol{p}_{1}^{\prime} \boldsymbol{p}_{2}^{\prime}\right) f\left(\boldsymbol{p}_{1}^{\prime}, \boldsymbol{p}_{2}^{\prime}\right)=0,
\end{aligned}
$$

where $p_{a 0}=\sqrt{m^{2}+\mathbf{p}_{a}^{2}}$ and $\mathcal{K}^{(2)}$ is a kernel. In the case of a confining interaction this kernel is singular and must be regularized; see [6] and refs. therein.

In the case of the $\varphi^{3}$-nonlinear dipole model $\mathcal{L}_{\text {int }}^{(3)}$ and thus $H_{\text {int }}^{(3)}$ are trilinear in $\rho$. Consequently, the matrix element $\left\langle 2\left|H_{\text {int }}^{(3)}\right| 2\right\rangle$ and $\left\langle 1+\overline{1}\left|H_{\text {int }}^{(3)}\right| 1+\overline{1}\right\rangle$ vanish, similarly to the case of the nonlinear the Wick-Cutkosky model considered in [7]. In other words, the purely two-particle trial states, $|2\rangle$ and $|1+\overline{1}\rangle$, are inadequate for describing bound states, as they do not sample the interaction terms of this Hamiltonian. In contrast, the three-particle states are affected by $H_{\text {int }}^{(3)}$, and the (non trivial) matrix element $\left\langle 3\left|H_{\text {int }}^{(3)}\right| 3\right\rangle$ leads to the tree-particle wave equation:

$$
\begin{aligned}
& \left\{p_{10}+p_{20}+p_{30}-E\right\} f\left(\boldsymbol{p}_{1}, \boldsymbol{p}_{2}, \boldsymbol{p}_{3}\right) \\
& +\int \mathrm{d}^{3} p_{1}^{\prime} \mathrm{d}^{3} p_{2}^{\prime} \mathrm{d}^{3} p_{3}^{\prime} \mathcal{K}^{(3)}\left(\boldsymbol{p}_{1}, \boldsymbol{p}_{2}, \boldsymbol{p}_{3}, \boldsymbol{p}_{1}^{\prime}, \boldsymbol{p}_{2}^{\prime}, \boldsymbol{p}_{3}^{\prime}\right) \\
& \times f\left(\boldsymbol{p}_{1}^{\prime}, \boldsymbol{p}_{2}^{\prime}, \boldsymbol{p}_{3}^{\prime}\right)=0 .
\end{aligned}
$$

The kernel $\mathcal{K}^{(3)}$ of this equation is rather complex [7] and is expected to be singular (at least for a massless mediating field). The study of this question is a subtle problem, as is the problem of regularization of the kernel $\mathcal{K}^{(3)}$. In particular, it should be regularized in accord with the regularization of the non-relativistic potential (4.9) considered in Section 4. In view of this, another question arises: do QFT-counterparts of pairwise interactions which are present in the nonrelativistic potentials (5.3) and (5.4) exist? If yes, they would not be sampled by the purely two particle trial states $|2\rangle$ and $|1+\overline{1}\rangle$. A possible solution to this problem is to use more general trial states of the form $\left|\Psi_{\mathrm{tr}}\right\rangle=|2\rangle+|4\rangle$, $\left|\Psi_{\mathrm{tr}}\right\rangle=|1+\overline{1}\rangle+|2+\overline{2}\rangle$ etc. $[20]$.

\section{APPENDIX. CALCULATING $F_{-++}$AND $F_{+++}$}

Combining definitions (4.4), (4.3) and (2.8) yields the following expression for the function $F_{-++}(u, v)$ :

$$
F_{-++}(u, v)=\frac{1}{(2 \pi)^{3}} \int \mathrm{d}^{4} z \theta\left(u_{0}-z_{0}\right) \delta\left[(z-u)^{2}\right] \theta\left(z_{0}-v_{0}\right) \delta\left[(z-v)^{2}\right] \theta\left(z_{0}\right) \delta\left(z^{2}\right) .
$$




\section{A. DUVIRYAK, J. W. DAREWYCH}

In order to evaluate this integral we consider different cases.

I.1) Let $u$ be time-like, i.e., $u^{2}>0$. Due to the Lorentz-invariance of the function $F_{-++}(u, v)$ one can choose a reference frame in which $\boldsymbol{u}=0$. Then the following transformations in the integrand of (A.1) are useful:

$$
\begin{aligned}
& \theta\left(z_{0}\right) \delta\left(z^{2}\right)=\frac{\delta\left(z_{0}-|\boldsymbol{z}|\right)}{2|\boldsymbol{z}|} ; \\
& \theta\left(u_{0}-z_{0}\right) \stackrel{(\mathrm{A} .2)}{=} \theta\left(u_{0}-|\boldsymbol{z}|\right) ; \\
& \delta\left[\left(u_{0}-z_{0}\right)^{2}-\boldsymbol{z}^{2}\right] \stackrel{(\mathrm{A} .2)}{=} \delta\left(u_{0}^{2}-2 u_{0}|\boldsymbol{z}|\right)=\frac{\delta\left(|\boldsymbol{z}|-u_{0} / 2\right)}{2 u_{0}} ; \\
& \theta\left(z_{0}-v_{0}\right) \stackrel{(\mathrm{A} .4)}{=} \theta\left(u_{0}-2 v_{0}\right) ; \\
& \delta\left[(z-v)^{2}\right] \stackrel{(\mathrm{A} .2)}{=} \delta\left(v^{2}-2 z \cdot v\right) \stackrel{(\mathrm{A} .4)}{=} \delta\left[u_{0}\left(|\boldsymbol{v}| k-v_{0}\right)+v^{2}\right]=\frac{1}{u_{0}|\boldsymbol{v}|} \delta\left(k+\frac{v^{2}-u_{0} v_{0}}{u_{0}|\boldsymbol{v}|}\right), \\
& \text { where } k=\frac{\boldsymbol{z} \cdot \boldsymbol{v}}{|\boldsymbol{z}||\boldsymbol{v}|} .
\end{aligned}
$$

Since $|k| \leq 1$ then, by (A.6),

$$
\left|v^{2}-u_{0} v_{0}\right|-u_{0}|\boldsymbol{v}| \leq 0
$$

otherwise the integrand in (A.1) vanishes.

I.1.1a) Let $v^{2}>0, v_{0}>|\boldsymbol{v}|$. Then, by (A.4), $v^{2}-u_{0} v_{0}<-v_{0}^{2}-\boldsymbol{v}^{2}<0$. The l.h.s. of inequality (A.8) can be expressed, using (A.5), as follows:

$$
u_{0}\left(v_{0}-|\boldsymbol{v}|\right)-v^{2}=\left(u_{0}-v_{0}-|\boldsymbol{v}|\right)\left(v_{0}-|\boldsymbol{v}|\right)>\left(v_{0}-|\boldsymbol{v}|\right)^{2}>0
$$

This inequality is opposite to (A.8). Thus $F_{++}(u, v)=0$ in this case.

I.1.1b) Let $v^{2}>0, v_{0}<-|\boldsymbol{v}|$. Then $v^{2}-u_{0} v_{0}>v^{2}+u_{0}|\boldsymbol{v}|>0$. The l.h.s. of inequality (A.8) becomes: $v^{2}-u_{0}\left(v_{0}+\right.$ $|\boldsymbol{v}|)>v^{2}>0$. Thus $F_{++}(u, v)=0$ in this case.

I.1.2) Let $v^{2}<0$. We replace the inequality (A.8) by an equivalent one:

$$
\left(v^{2}-u_{0} v_{0}\right)^{2}-u_{0} \boldsymbol{v}^{2}=v^{2}\left(v^{2}-2 u_{0} v_{0}+u_{0}^{2}\right) \leq 0
$$

which then simplifies to the condition

$$
\left(v_{0}-u_{0}\right)^{2}-\boldsymbol{v}^{2} \geq 0
$$

If this condition holds, the integral (A.1) does not vanish and can be evaluated directly, using (A.2)-(A.7) and spherical coordinates:

$$
\begin{aligned}
F_{-++}(u, v)=\frac{1}{(2 \pi)^{3}} \int_{0}^{2 \pi} \mathrm{d} \varphi \int_{-1}^{1} \mathrm{~d} k \int_{0}^{\infty}|\boldsymbol{z}| \mathrm{d}|\boldsymbol{z}| & \int_{-\infty}^{\infty} \mathrm{d} z_{0} \frac{\delta\left(z_{0}-|\boldsymbol{z}|\right)}{2|\boldsymbol{z}|} \\
& \times \frac{\delta\left(|\boldsymbol{z}|-u_{0} / 2\right)}{2 u_{0}} \frac{1}{u_{0}|\boldsymbol{v}|} \delta\left(k+\frac{v^{2}-u_{0} v_{0}}{u_{0}|\boldsymbol{v}|}\right)=\frac{1}{2(4 \pi)^{2} u_{0}|\boldsymbol{v}|} .
\end{aligned}
$$

Otherwise, $F_{-++}(u, v)=0$.

I.2) Let $u^{2}<0$. There exists a reference frame in which $u_{0}<0$. Then the integrand of (A.1) vanishes due to the factor $\theta\left(u_{0}-z_{0}\right) \theta\left(z_{0}\right)=0$.

Now we return to an arbitrary reference frame in which the function $F_{-++}(u, v)$ should be expressed in terms of Lorentz-invariant combinations of 4 -vectors $u$ and $v$. This can be done unambiguously, by the replacement:

$$
\left(u_{0}-v_{0}\right)^{2}-\boldsymbol{v}^{2} \mapsto(u-v)^{2}, \quad u_{0}|\boldsymbol{v}| \mapsto \sqrt{(u \cdot v)^{2}-u^{2} v^{2}} \quad \text { etc. }
$$

The result yields the form (4.5).

Upon calculating $F_{+++}(u, v)$ we note that it is described by the same integral as (A.1) but with the factor $\theta\left(u_{0}-z_{0}\right)$ replaced by $\theta\left(z_{0}-u_{0}\right)$. Besides, this function is symmetric: $F_{+++}(u, v)=F_{+++}(v, u)$. Thus it is sufficient to consider just two cases. 
II.1) Let $u^{2}>0$ or/and $v^{2}>0$. Using reasoning similar to that used in the case I.1 one can prove that $F_{+++}(u, v)=0$.

II.2) Let $u^{2}<0$ and $v^{2}<0$. One can choose the reference frame in which $u=\left(0, u_{1}, 0,0\right), v=\left(0, v_{1}, v_{2}, 0\right)$. Then

$$
\begin{aligned}
& \delta\left[(z-u)^{2}\right] \stackrel{(\mathrm{A} .2)}{=} \delta\left(2 z_{1} u_{1}-u_{1}^{2}\right)=\frac{\delta\left(z_{1}-u_{1} / 2\right)}{2 u_{1}} ; \\
& \delta\left[(z-v)^{2}\right] \stackrel{(\mathrm{A} .2)}{=} \delta\left(v^{2}-2 z \cdot v\right) \stackrel{(\mathrm{A} .12)}{=} \delta\left(2 v_{2} z_{2}+v_{1} u_{1}-v_{1}^{2}-v_{2}^{2}\right)=\frac{1}{2 v_{2}} \delta\left(z_{2}-\frac{v_{1}^{2}+v_{2}^{2}-u_{1} v_{1}}{2 v_{2}}\right),
\end{aligned}
$$

and thus

$$
\begin{gathered}
F_{+++}(u, v)=\frac{1}{(2 \pi)^{3}} \iiint \int \mathrm{d} z_{0} \mathrm{~d} z_{1} \mathrm{~d} z_{2} \mathrm{~d} z_{3} \frac{\delta\left(z_{0}-|\boldsymbol{z}|\right)}{2|\boldsymbol{z}|} \\
\times \frac{\delta\left(z_{1}-u_{1} / 2\right)}{2 u_{1}} \frac{1}{2 v_{2}} \delta\left(z_{2}-\frac{v_{1}^{2}+v_{2}^{2}-u_{1} v_{1}}{2 v_{2}}\right) \\
\quad=\frac{1}{(4 \pi)^{3}} \int_{-\infty}^{\infty} \frac{\mathrm{d} z_{3}}{u_{1} v_{2}}\left[\frac{u_{1}^{2}}{4}+\left(\frac{v_{1}^{2}+v_{2}^{2}-u_{1} v_{1}}{2 v_{2}}\right)^{2}+z_{3}^{2}\right]^{-1 / 2} .
\end{gathered}
$$

This last integral (A.14) is divergent.

The result is summarized in (4.6).

[1] J. Greensite, Prog. Part. Nucl. Phys. 51, 1 (2003).

[2] E. S. Swanson, AIP Conf. Proc. 717, 636 (2004).

[3] J. Kiskis, Phys. Rev. D 11, 2178 (1975).

[4] S. Blaha, Phys. Rev. D 10, 4268 (1975).

[5] A. I. Alekseev, B. A. Arbuzov, Theor. Math. Phys. 59, 372 (1984).

[6] A. Duviryak, J. W. Darewych, J. Phys. A 37, 8365 (2004).

[7] A. Duviryak, J. W. Darewych, J. Phys. A 43, 485402 (2010).

[8] W. Lucha, F. F. Schoberl, D. Gromes, Phys. Reports 200, 127 (1991).

[9] A. Inopin, G. S. Sharov, Phys. Rev. 63, 054023 (2001).

[10] A. D. Fokker, Z. Phys. 28, 386 (1929).

[11] P. Havas, In Problems in the Foundations of Physics,
M. Bunge, ed. (Springer-Verlag, Berlin-Heidelberg-New York, 1971), p. 31.

[12] A. Rivacoba, Nuovo Cimento B 8435 (1984).

[13] J. Weiss, J. Math. Phys. 27, 1015 (1986).

[14] A. Duviryak, Int. J. Mod. Phys. A 14, 4519 (1999).

[15] A. Duviryak, Int. J. Mod. Phys. A 16, 2771 (2001).

[16] A. Duviryak, Collected Phys. Papers of the Shevchenko Sci. Soc. 7, 533 (2008).

[17] J. Darewych, Condens. Matter Phys. 3, 633 (2000).

[18] B. Ding, J. Darewych, J. Phys. G 26, 97 (2000).

[19] M. Emami-Razavi, J. W. Darewych, J. Phys. G 31, 1095 (2005).

[20] M. Emami-Razavi, J. W. Darewych, J. Phys. G 32, 1171 (2006).

\title{
ПРО УТРИМУЮЧІ ВЗАЄМОДІЇ У СКАЛЯРНИХ УЗАГАЛЬНЕННЯХ ДИПОЛЬНОї МОДЕЛІ
}

\author{
А. Дувіряк ${ }^{1}$, Ю. Даревич ${ }^{2}$ \\ ${ }^{1}$ Відділ комп'ютерного моделювання багаточастинкових систем, \\ Інститут фізики конденсованих систем НАН України, \\ вул. Свенціцького, 1, Лъвів, 79011, Украӥна \\ ${ }^{2}$ Відділення фізики та астрономї, Йорксъкий університет, \\ Торонто, Онтаріо, МЗЈ 1Р3, Канада
}

\footnotetext{
Розглянуто дві модифіковані версії дипольної моделі з парою скалярних полів-посередників. Одна 3 модифікацій стосується юкавського члена в лагранжіані. В іншій версії додано нелінійний член самодії. Шляхом побудови точних розв'язків польових рівнянь полів-посередників у термінах коваріантних функцій Гріна отримано лагранжіан із часо-нелокальними багатоточковими членами взаємодії. У межах лінійної модифікованої дипольної моделі отримано двочастинкову взаємодію, що містить кулонівський внесок та лінійний конфайнмент. Нелінійна $\varphi^{3}$-модель приводить до парної та тричастинкової кластерної взаємодій із логарифмічним конфайнментом. Запропоновано шляхи послідовного релятивістського тлумачення моделей та обговорено проблему розбіжностей.
} 